\title{
AN APPROXIMATION THEORY FOR FOCAL POINTS AND FOCAL INTERVALS ${ }^{1}$
}

\author{
JOHN GREGORY
}

\begin{abstract}
The theory of focal points and conjugate points is an important part of the study of problems in the calculus of variations and control theory. In previous works we gave a theory of focal points and of focal intervals for an elliptic form $J(x)$ on a Hilbert space $\mathscr{A}$. These results were based upon inequalities dealing with the indices $s(\sigma)$ and $n(\sigma)$ of the elliptic form $J(x ; \sigma)$ defined on the closed subspace $\mathscr{A}(\sigma)$ of $\mathscr{A}$, where $\sigma$ belongs to the metric space $(\Sigma, \rho)$.

In this paper we give an approximation theory for focal point and focal interval problems. Our results are based upon inequalities dealing with the indices $s(\mu)$ and $u(\mu)$, where $\mu$ belongs to the metric space $(M, d), M=E^{1} \times \Sigma$. For the usual focal point problems we show that $\lambda_{n}(\sigma)$, the $n$th focal point, is a $\rho$ continuous function of $\sigma$. For the focal interval case we give sufficient hypotheses so that the number of focal intervals is a local minimum at $\sigma_{0}$ in $\Sigma$. Neither of these results seems to have been published before (under any setting) in the literature. For completeness an example is given for quadratic problems in a control theory setting.
\end{abstract}

1. Preliminaries. We now state the approximation hypothesis given in [1] and [2] and the focal point hypothesis given in [5]. The former is contained in conditions (1) and (2), the latter in (3). $\mathscr{A}$ will denote a real Hilbert space with inner product $(x, y)$ and norm $\|x\|=(x, x)^{\mathbf{1 / 2}}$. Strong convergence is denoted by $x_{q} \Rightarrow x_{0}$ and weak convergence by $x_{q} \rightarrow x_{0}$.

Let $\Sigma$ be a metric space with metric $\rho$. A sequence $\left\{\sigma_{r}\right\}$ in $\Sigma$ converges to $\sigma_{0}$ in $\Sigma$, written $\sigma_{r} \rightarrow \sigma_{0}$, if $\lim _{r \Rightarrow \infty} \rho\left(\sigma_{r}, \sigma_{0}\right)=0$. For each $\sigma$ in $\Sigma$ let $\mathscr{A}(\sigma)$ be a closed subspace of $\mathscr{A}$ such that

(1a) if $\sigma_{r} \rightarrow \sigma_{0}, x_{r}$ in $\mathscr{A}\left(\sigma_{r}\right), x_{r} \rightarrow y_{0}$ then $y_{0}$ is in $\mathscr{A}\left(\sigma_{0}\right)$;

(1b) if $x_{0}$ is in $\mathscr{A}\left(\sigma_{0}\right)$ and $\varepsilon>0$ there exists $\delta>0$ such that whenever $\rho\left(\sigma, \sigma_{0}\right)<\delta$, there exists $x_{\sigma}$ in $A(\sigma)$ satisfying $\left\|x_{0}-x_{\sigma}\right\|<\varepsilon$.

Received by the editors March 26, 1971.

AMS 1969 subject classifications. Primary 4160, 4617; Secondary 49XX, 9340.

Key words and phrases. Approximation theory, focal points, conjugate points, calculus of variations, control theory, Hilbert space, quadratic forms.

${ }^{1}$ The preparation of this paper was sponsored in part by the U.S. Army Research Office under Grant DA-31-124-ARO(D)-355. Reproduction in whole or in part is permitted for any purpose of the United States Government.

(C) American Mathematical Society 1972 
For each $\sigma$ in $\Sigma$ let $J(x ; \sigma)$ be a quadratic form defined on $\mathscr{A}(\sigma)$ with $J(x, y ; \sigma)$ the associated bilinear form. For $r=0,1,2, \cdots$, let $x_{r}$ be in $\mathscr{A}\left(\sigma_{r}\right), y_{r}$ in $\mathscr{A}\left(\sigma_{r}\right)$ such that if $x_{r} \rightarrow x_{0}, y_{r} \Rightarrow y_{0}$ and $\sigma_{r} \rightarrow \sigma_{0}$ then

(2a) $\lim _{r \rightarrow \infty} J\left(x_{r}, y_{r} ; \sigma_{r}\right)=J\left(x_{0}, y_{0} ; \sigma_{0}\right)$;

(2b) $\lim _{\inf _{r \rightarrow \infty}} J\left(x_{r} ; \sigma_{r}\right) \geqq J\left(x_{0} ; \sigma_{0}\right)$; and

(2c) $\lim _{r \rightarrow \infty} J\left(x_{r} ; \sigma_{r}\right)=J\left(x_{0} ; \sigma_{0}\right)$ implies $x_{r} \Rightarrow x_{0}$.

Let $a, b$ be real numbers $(a<b)$ and define $\Lambda=[a, b]$. Let $\{\mathscr{H}(\lambda): \lambda$ in $\Lambda\}$ be a one parameter family of closed subspaces of $\mathscr{A}$ such that $\mathscr{H}(a)=0$, $\mathscr{H}(b)=\mathscr{A}$, and $\mathscr{H}\left(\lambda_{1}\right) \subset \mathscr{H}\left(\lambda_{2}\right)$ whenever $\lambda_{1}, \lambda_{2}$ in $\Lambda, \lambda_{1}<\lambda_{2}$. In this paper we will require one or both of the additional hypotheses:

(3a) $\mathscr{H}\left(\lambda_{0}\right)=\bigcap_{\lambda_{0}<\lambda \leqq b} \mathscr{H}(\lambda)$ whenever $a \leqq \lambda_{0}<b$, and

(3b) $\mathscr{H}\left(\lambda_{0}\right)=\operatorname{cl}\left(\bigcup_{a \leqq \lambda<\lambda_{0}} \mathscr{H}(\lambda)\right)$ whenever $a<\lambda_{0} \leqq b$ is satisfied. We note that $\mathrm{cl} S$ denotes the closure of $S$.

THEOREM 1. Condition (3a) implies (1a) holds in the $\mathscr{H}, \lambda$ notation; (3b) implies (1b) holds in the $\mathscr{H}, \lambda$ notation. Finally (3) implies (1) holds in the $\mathscr{H}, \lambda$ notation.

This result has been given in [1].

The signature (index) of a bilinear form $Q(x)$ on a subspace $\mathscr{B}$ of $\mathscr{A}$ is the dimension of a maximal, linear subclass $\mathscr{C}$ of $\mathscr{B}$ such that $x \neq 0$ in $\mathscr{C}$ implies $Q(x)<0$. The nullity of $Q(x)$ on $\mathscr{B}$ is the dimension of the set $\mathscr{B}_{0}=\{x$ in $\mathscr{B} \mid Q(x, y)=0$ for all $y$ in $\mathscr{B}\}$. The vector $x$ is said to be a $Q$ null vector of $B$. For Theorem 2 we denote the index and nullity of $J(x ; \sigma)$ on $\mathscr{A}(\sigma)$ by $s(\sigma)$ and $n(\sigma)$.

Theorem 2. Conditions (1a), (2b) and (2c) imply there exists $\delta>0$ such that $\rho\left(\sigma, \sigma_{0}\right)<\delta$ implies $s(\sigma)+n(\sigma) \leqq s\left(\sigma_{0}\right)+n\left(\sigma_{0}\right)$. Conditions $(1 \mathrm{~b})$ and (2a) imply there exists $\delta>0$ such that $\rho\left(\sigma, \sigma_{0}\right)<\delta$ implies $s\left(\sigma_{0}\right) \leqq s(\sigma)$.

This result has been given in [1].

2. Approximation theory. We now define the spaces $\mathscr{B}(\mu)$ which "resolve" the space $\mathscr{A}(\sigma)$. Inequality results are then given relating the signatures $s(\mu)$ and $n(\mu)$ to $s\left(\mu_{0}\right)$ and $n\left(\mu_{0}\right)$.

Let $M=\Lambda \times \Sigma$ be the metric space with metric $d$ defined by $d\left(\mu_{1}, \mu_{2}\right)=$ $\left|\lambda_{2}-\lambda_{1}\right|+\rho\left(\sigma_{2}, \sigma_{1}\right)$ where $\mu_{1}=\left(\lambda_{1}, \sigma_{1}\right)$ and $\mu_{2}=\left(\lambda_{2}, \sigma_{2}\right)$. For each $\mu=(\lambda, \sigma)$ in $M$ define $J(x ; \mu)=J(x ; \sigma)$ on the space $\mathscr{B}(\mu)=\mathscr{A}(\sigma) \cap \mathscr{H}(\lambda)$. Let $s(\mu)=s(\lambda, \sigma), n(\mu)=n(\lambda, \sigma)$ denote the index and nullity of $J(x ; \mu)$ on $\mathscr{B}(\mu)$.

We will use the terminology "holds on $M$ " to refer to conditions (1) and (2) in the " $\mu$ setting" of this section as opposed to the " $\sigma$ setting" of $\S 1$. Lemma 3 is immediate as $J(x ; \mu)=J(x ; \sigma)$ on $\mathscr{A}(\mu)$.

LEMMA 3. If (2) holds on $\Sigma$ then (2) holds on $M$. 
LEMMA 4. If (1a) holds on $\Sigma$ and (3a) holds, then (1a) holds on $M$.

Suppose $\mu_{q} \rightarrow \mu_{0}, x_{q}$ in $\mathscr{B}\left(\mu_{q}\right), x_{q} \rightarrow x_{0}$, where $\mu_{q}=\left(\lambda_{q}, \sigma_{q}\right), q=0,1,2, \cdots$. From $\sigma_{q} \rightarrow \sigma_{0}, x_{q}$ in $\mathscr{A}\left(\sigma_{q}\right), x_{q} \rightarrow x_{0}$ we have $x_{0}$ in $\mathscr{A}\left(\sigma_{0}\right)$. From $\lambda_{q} \rightarrow \lambda_{0}$ and Theorem 1 we have $x_{0}$ in $\mathscr{H}\left(\lambda_{0}\right)$. Thus $x_{0}$ in $\mathscr{H}\left(\lambda_{0}\right) \cap \mathscr{A}\left(\sigma_{0}\right)=\mathscr{B}\left(\mu_{0}\right)$.

THEOREM 5. Assume (1a) and (2) hold on $\Sigma$ and that (3a) holds. For any $\mu_{0}=\left(\lambda_{0}, \sigma_{0}\right)$ in $M$ there exists $\delta>0$ such that if $\mu=(\lambda, \sigma), d\left(\mu_{0}, \mu\right)<\delta$ then

$$
s(\lambda, \sigma)+n(\lambda, \sigma) \leqq s\left(\lambda_{0}, \sigma_{0}\right)+n\left(\lambda_{0}, \sigma_{0}\right) .
$$

Lemmas 3 and 4 imply that the hypothesis of the first statement of Theorem 2 holds on $M$. Inequality (4) is the first conclusion of Theorem 2 in this notation.

We note that ( $1 \mathrm{~b}$ ) does not hold on $M$ without extra hypotheses. This is due to the fact that the $x_{\mu}$ which satisfies $\left\|x_{0}-x_{\mu}\right\|<\varepsilon$ may belong to both $\mathscr{H}(\lambda)$ and $\mathscr{A}(\sigma)$. Fortunately these extra hypotheses are not necessary to prove inequality (5).

Theorem 6. Assume (1b) and (2) hold on $\Sigma$ and that (3b) holds. For any $\mu_{0}=\left(\lambda_{0}, \sigma_{0}\right)$ in $M$ there exists $\delta>0$ such that if $\mu=(\lambda, \sigma), d\left(\mu_{0}, \mu\right)<\delta$ then

$$
s\left(\lambda_{0}, \sigma_{0}\right) \leqq s(\lambda, \sigma) .
$$

We note there exists $\delta>0$ such that $d\left(\mu_{0}, \mu\right)<\delta$ implies the following inequalities hold:

$$
s\left(\lambda_{0}, \sigma_{0}\right) \leqq s\left(\lambda_{0}-\delta, \sigma_{0}\right) \leqq s\left(\lambda_{0}-\delta, \sigma\right) \leqq s(\lambda, \sigma) .
$$

The first inequality holds by the second conclusion of Theorem 2 as

$$
\mathscr{B}\left(\lambda_{0}, \sigma_{0}\right)=\operatorname{cl}\left(\bigcup_{a \leqq \lambda<\lambda_{0}} \mathscr{B}\left(\lambda, \sigma_{0}\right)\right) \quad \text { whenever } a<\lambda_{0} \leqq b .
$$

The second inequality holds by replacing $\mathscr{A}$ with $\mathscr{H}\left(\lambda_{0}-\sigma\right)$ in (1b). More specifically if $\hat{x}$ is the projection of $x$ onto $\mathscr{H}(\lambda-0)$ and $x_{\sigma}$ is in $\mathscr{A}(\sigma)$ and given by (1b) then $\hat{x}_{\sigma}$ in $\mathscr{H}(\lambda-0) \cap \mathscr{A}(\sigma)$ and $\left\|\hat{x}_{\sigma}-x_{0}\right\| \leqq$ $\left\|x_{\sigma}-x_{0}\right\|<\varepsilon$. The third inequality follows as $\mathscr{H}\left(\lambda_{0}-\delta\right) \subset \mathscr{H}(\lambda)$.

Combining Theorems 5 and 6 we have:

TheOREM 7. For any $\mu_{0}=\left(\lambda_{0}, \sigma_{0}\right)$ in $M$ there exists $\delta>0$ such that if $\mu=(\lambda, \sigma), d\left(\mu_{0}, \mu\right)<\delta$ then

(6) $s\left(\lambda_{0}, \sigma_{0}\right) \leqq s(\lambda, \sigma) \leqq s(\lambda, \sigma)+n(\lambda, \sigma) \leqq s\left(\lambda_{0}, \sigma_{0}\right)+n\left(\lambda_{0}, \sigma_{0}\right)$.

\section{Furthermore}

$$
n\left(\lambda_{0}, \sigma_{0}\right)=0 \text { implies } s(\lambda, \sigma)=s\left(\lambda_{0}, \sigma_{0}\right) \text { and } n(\lambda, \sigma)=0 .
$$


3. Focal points and focal intervals. Let $\sigma_{0}$ in $\Sigma$ be given. A point $\lambda_{0}$ at which $s\left(\lambda, \sigma_{0}\right)$ is discontinuous will be called a focal point of $J\left(x ; \sigma_{0}\right)$ relative to $\{\mathscr{H}(\lambda): \lambda$ in $\Lambda\}$. The difference $s\left(\lambda_{0}+0, \sigma_{0}\right)-s\left(\lambda_{0}, \sigma_{0}\right)$ will be called the order of $\lambda_{0}$ as a focal point (of $\sigma_{0}$ ). A focal point $\lambda_{0}$ is counted the number of times equal to its order. In the above $s\left(\lambda_{0}+0, \sigma_{0}\right)$ is the right-hand limit of $s\left(\lambda, \sigma_{0}\right)$ as $\lambda \rightarrow \lambda_{0}$ from above. The quantity $s\left(\lambda_{0}-0, \sigma_{0}\right)$ is similarly defined.

It has been shown in [1] and [5] that (3b) implies $s\left(\lambda-0, \sigma_{0}\right)=s\left(\lambda, \sigma_{0}\right)$ while (3a) and the disjoint hypotheses of Theorem 8 imply $s\left(\lambda+0, \sigma_{0}\right)=$ $s\left(\lambda, \sigma_{0}\right)+n\left(\lambda, \sigma_{0}\right)$. Thus

THEOREM 8. Assume (3) holds. Let $\sigma_{0}$ in $\Sigma$ be given such that $\lambda^{\prime}, \lambda^{\prime \prime}$ in $\Lambda, a \leqq \lambda^{\prime}<\lambda^{\prime \prime} \leqq b$ imply the $J\left(x ; \sigma_{0}\right)$ null vectors on $\mathscr{B}\left(\lambda^{\prime}, \sigma_{0}\right)$ and $\mathscr{B}\left(\lambda^{\prime \prime}, \sigma_{0}\right)$ are disjoint. Assume $\lambda^{\prime}$ and $\lambda^{\prime \prime}$ are not focal points of $\sigma_{0}\left(a \leqq \lambda^{\prime}<\lambda^{\prime \prime}<b\right)$ and there exist $k$ focal points of $\sigma_{0}$ on $\left(\lambda^{\prime}, \lambda^{\prime \prime}\right)$. Then there exists $\varepsilon>0$ such that $\rho\left(\sigma, \sigma_{0}\right)<\varepsilon$ implies there are exactly $k$ focal points of $\sigma$ on $\left(\lambda^{\prime}, \lambda^{\prime \prime}\right)$.

In fact if $\lambda_{n}\left(\sigma_{0}\right) \leqq \lambda_{n+1}\left(\sigma_{0}\right) \leqq \cdots \leqq \lambda_{n+k-1}\left(\sigma_{0}\right) \quad(n=1,2,3, \cdots)$ are the $k$ focal points of $\sigma_{0}$ on $\left(\lambda^{\prime}, \lambda^{\prime \prime}\right)$ then $\lambda_{n}(\sigma) \leqq \lambda_{n+1}(\sigma) \leqq \cdots \leqq \lambda_{n+k-1}(\sigma)$ are the $k$ focal points of $\sigma$ on $\left(\lambda^{\prime}, \lambda^{\prime \prime}\right)$.

Assume $s\left(\lambda^{\prime}, \sigma_{0}\right)=n$. Then by the above remark, $s\left(\lambda^{\prime \prime}, \sigma_{0}\right)=n+k-1$ and $n\left(\lambda^{\prime}, \sigma_{0}\right)=n\left(\lambda^{\prime \prime}, \sigma_{0}\right)=0$. By (7) there exists $\delta>0$ such that if $\rho\left(\sigma, \sigma_{0}\right)<\delta$ then $n\left(\lambda^{\prime}, \sigma\right)=n\left(\lambda^{\prime \prime}, \sigma\right)=0, s\left(\lambda^{\prime}, \sigma\right)=n, s\left(\lambda^{\prime \prime}, \sigma\right)=n+k-1$. The result follows by definition.

COROLlaRy 9. Under the above hypotheses there exists $\varepsilon>0$ such that $\rho\left(\sigma, \sigma_{0}\right)<\varepsilon$ and $a \leqq \lambda \leqq a+\varepsilon$ imply there exists no focal point $\lambda$ of $\sigma$.

COROLlary 10. Under the above hypotheses the nth focal point $\lambda_{n}(\sigma)$ is a continuous function of $\sigma(n=1,2,3, \cdots)$.

If we assume that the disjoint hypotheses of Theorem 8 do not hold we obtain a focal interval theory. In this case condition (3) implies that if $x_{0}$ is a $J\left(x ; \sigma_{0}\right)$ null vector of $\mathscr{B}\left(\lambda_{0}, \sigma_{0}\right)$ then $\sigma_{0}$ belongs to a proper closed subinterval $\Lambda_{1}$ of $\Lambda$ where $\Lambda_{1}=\left\{\lambda\right.$ in $\Lambda: x_{0}$ is a $J\left(x ; \sigma_{0}\right)$ null vector of $\left.\mathscr{B}\left(\lambda, \sigma_{0}\right)\right\}$. [2] shows that focal intervals can be well defined, and contain the relationship between focal intervals and the indices $s(\lambda, \sigma)$ and $n(\lambda, \sigma)$.

Very briefly let $\sigma_{0}$ be in $\Sigma$ and assume $\lambda_{1}$ is the first focal point (with respect to $\sigma_{0}$ ) with order $e_{1}=e_{1}\left(\sigma_{0}\right)$. The first $e_{1}$ focal intervals $I_{1}\left(\sigma_{0}\right), \cdots$, $I_{e_{1}}\left(\sigma_{0}\right)$ end at $\lambda_{1}$. They are closed intervals whose left-hand endpoint $\lambda_{j 1}\left(\sigma_{0}\right)$ is given recursively for $j=1, \cdots, e_{1}$ by

$$
\lambda_{j 1}\left(\sigma_{0}\right)=\min \left\{\lambda \leqq \lambda_{1}: \text { there exists } x \neq 0 \text { in } S_{j}\right\}
$$

where $S_{j}$ is the set of $J\left(x ; \sigma_{0}\right)$ null vectors of $\mathscr{B}\left(\lambda_{1}, \sigma_{0}\right)$ which are not 
$J\left(x ; \sigma_{0}\right)$ null vectors of $\mathscr{B}\left(\lambda_{1}+0, \sigma_{0}\right)$, such that $\left(x_{j}, x_{k}\right)=0$ for $k=1, \cdots$, $j-1$, where $x_{j}$ is the vector "giving" $\lambda_{j 1}$.

With obvious modifications, the remaining focal intervals may be defined corresponding to the distinct focal points $\lambda_{1}<\lambda_{2}<\cdots<\lambda_{p}$. Note that $s\left(\lambda, \sigma_{0}\right)$ equals the number of focal intervals on the open interval $(a, \lambda)$.

In the remainder of this section we will consider inequalities involving $f\left(\lambda^{\prime}, \lambda^{\prime \prime} ; \sigma\right)$, the number of focal intervals (with respect to $\sigma$ ) on the interval $\left(\lambda^{\prime}, \lambda^{\prime \prime}\right)$ of $\Lambda$. We will denote the dimension of the $J(x ; \sigma)$ null vectors common to the space $\mathscr{B}\left(\lambda^{\prime}, \sigma\right)$ and $\mathscr{B}\left(\lambda^{\prime \prime}, \sigma\right)$ by $m\left(\lambda^{\prime}, \lambda^{\prime \prime}, \sigma\right)$. Theorem 11 has been given in [2].

Theorem 11. Let $\sigma_{0}$ in $\Sigma$. If $\lambda^{\prime}, \lambda^{\prime \prime}$ in $\Lambda\left(a \leqq \lambda^{\prime}<\lambda^{\prime \prime}<b\right)$ then

(8) $f\left(\lambda^{\prime}, \lambda^{\prime \prime} ; \sigma_{0}\right)=s\left(\sigma^{\prime \prime} ; \sigma_{0}\right)-\left[s\left(\lambda^{\prime}, \sigma_{0}\right)+n\left(\lambda^{\prime}, \sigma_{0}\right)\right]+m\left(\lambda^{\prime}, \lambda^{\prime \prime} ; \sigma_{0}\right)$.

THEOREM 12. Let $\lambda^{\prime}, \lambda^{\prime \prime}$ in $\Lambda\left(a \leqq \lambda^{\prime}<\lambda^{\prime \prime}<b\right)$; $\eta>0$; and assume $\sigma$ in $\Sigma, \quad \rho\left(\sigma_{0}, \sigma\right)<\eta$ implies $m\left(\lambda^{\prime}, \lambda^{\prime \prime} ; \sigma_{0}\right) \leqq m\left(\lambda^{\prime}, \lambda^{\prime \prime} ; \sigma\right)$. Then there exists $\delta>0$ such that $f\left(\lambda^{\prime}, \lambda^{\prime \prime} ; \sigma_{0}\right) \leqq f\left(\lambda^{\prime}, \lambda^{\prime \prime} ; \sigma\right)$ whenever $\rho\left(\sigma_{0}, \sigma\right)<\delta$.

From inequality (4) and equality (8) we have

$$
\begin{aligned}
f\left(\lambda^{\prime}, \lambda^{\prime \prime} ; \sigma_{0}\right) & =s\left(\lambda^{\prime \prime}, \sigma_{0}\right)-\left[s\left(\lambda^{\prime \prime}, \sigma_{0}\right)+n\left(\lambda^{\prime \prime}, \sigma_{0}\right)\right]+m\left(\lambda^{\prime}, \lambda^{\prime \prime} ; \sigma_{0}\right) \\
& \leqq s\left(\lambda^{\prime \prime}, \sigma\right)-\left[s\left(\lambda^{\prime \prime}, \sigma\right)+n\left(\lambda^{\prime \prime}, \sigma\right)\right]+m\left(\lambda^{\prime}, \lambda^{\prime \prime} ; \sigma\right) \\
& =f\left(\lambda^{\prime}, \lambda^{\prime \prime} ; \sigma\right) .
\end{aligned}
$$

Corollary 13. If $n\left(\lambda^{\prime \prime}, \sigma_{0}\right)=0$ then there exists $\delta>0$ such that $f\left(\lambda^{\prime}, \lambda^{\prime \prime} ; \sigma_{0}\right) \leqq f\left(\lambda^{\prime}, \lambda^{\prime \prime} ; \sigma\right)$ whenever $\rho\left(\sigma_{0}, \sigma\right)<\delta$.

In this case $n\left(\lambda^{\prime \prime}, \sigma\right)=0$ so that $m\left(\lambda^{\prime}, \lambda^{\prime \prime} ; \sigma_{0}\right)=0=m\left(\lambda^{\prime}, \lambda^{\prime \prime} ; \sigma\right)$.

4. An example. For our example we will consider a problem inspired by [6], which is the "modern day" control theory version of that of [4]. Further results for this example may be found in [2]. In [3] a further example is given in which the arcs $x(t)$ are broken line segments and the spaces $\mathscr{A}(\sigma)$ are finite dimensional.

An element $x$ of $\mathscr{A}$ is an $\operatorname{arc} x: x^{i}(t), u^{k}(t)(a \leqq t \leqq b)(i=1, \cdots, n$; $k=1, \cdots, q)$ where $x^{i}(t)$ and $u^{k}(t)$ are Lebesgue square integrable functions. The subspace $\mathscr{B}$ of $\mathscr{A}$ will denote all arcs which also satisfy:

$$
\dot{x}=A x+B u \text { and } C^{*} x(a)=0 .
$$

Finally $\mathscr{C}$ will denote all arcs $x$ in $\mathscr{B}$ which also satisfy $x(b)=0$.

The quadratic forms

$$
J(x ; \sigma)=x^{*}(a) D_{\sigma} x(a)+\int_{a}^{b} 2 \omega_{\sigma}(t, x, u) d t
$$


are assumed elliptic relative to the inner product

where

$$
(x, y)=x^{*}(a) y(a)+\int_{a}^{b}\left(y^{*} x+v^{*} u\right) d t
$$

and

$$
x: x(t), u(t) ; \quad y: y(t), v(t)
$$

$$
2 \omega_{\sigma}(t, x, u)=x^{*} P_{\sigma} x+x^{*} Q_{\sigma} u+u^{*} Q_{\sigma}^{*} x+u^{*} R_{\sigma} u .
$$

In the above let "**" denote the transpose of a matrix. The matrices $A, B, C$, and $D$ are respectively $n \times n, n \times q, n \times r$ and $n \times n$ constant real matrices where the rank of $C$ is $r \leqq n ; P_{\sigma}(t)$ and $Q_{\sigma}(t)$ are $n \times n$ and $n \times q$ Lebesgue square integrable matrices on $[a, b]$ with $P_{\sigma}(t)=P_{\sigma}^{*}(t)$; and $R_{\sigma}(t)=R_{\sigma}^{*}(t)$ is a $q \times q$ essentially bounded and Lebesgue integrable matrix on $[a, b]$ satisfying $R_{\sigma}(t) \geqq \varepsilon I$ almost everywhere for some $\varepsilon>0$. The ellipticity of $J$ is a consequence of the fact that $R_{\sigma}$ is positive definite in this sense.

For each $\lambda$ in $[a, b]$ let $\mathscr{C}(\lambda)$ be given by $\mathscr{C}(\lambda)=\{x$ in $\mathscr{C}: x(t)=0, u(t)=0$ a.e. on $\lambda \leqq t \leqq b\}$. Let $s(\lambda ; \sigma)$ and $n(\lambda ; \sigma)$ denote the signature and nullity of $J(x ; \sigma)$ on $\mathscr{C}(\lambda)$. We note that $\lambda_{1}<\lambda_{2}$ implies $\mathscr{C}\left(\lambda_{1}\right) \subset \mathscr{C}\left(\lambda_{2}\right)$ and that (3) holds with $\mathscr{C}$ and $\mathscr{C}(\lambda)$ replacing $\mathscr{A}$ and $\mathscr{H}(\lambda)$ respectively.

For fixed $\sigma$ the difference between the usual focal point phenomena and focal interval phenomena is the concept of abnormality. In the latter case a nonzero solution of Euler's equation (satisfying the transversality conditions) is allowed to equal zero on a subinterval of $[a, b]$. This is impossible in the former case. Mikami [6] has shown that if the matrices $A$ and $B$ are analytic in $[a, b]$ then for such solutions, $x(t)=0$ on some proper subinterval $\left[a^{\prime}, b^{\prime}\right]$ of $[a, b]$ implies $x(t)=0, u(t)=0$ a.e. on $[a, b]$. Thus all focal intervals degenerate to focal points in this case.

Continuity conditions (with respect to $\sigma$ ) on the matrices $D_{\sigma}, P_{\sigma}, Q_{\sigma}$ and $R_{\sigma}$ such that conditions (2) hold are left to the reader. The methods used in [3] will suffice to justify the more obvious cases. More advanced problems may be constructed by assuming matrices $A_{\sigma}, B_{\sigma}$, and $C_{\sigma}$ are indexed by $\sigma$, in which case we have the obvious changes of $\mathscr{B}(\sigma)$, instead of $\mathscr{B}$ and $s(\lambda, \sigma)$ and $n(\lambda, \sigma)$ as the signature and nullity of $J(x ; \sigma)$ on $\mathscr{B}(\sigma) \cap \mathscr{C}(\lambda)$.

\section{REFERENCES}

1. J. Gregory, An approximation theory for elliptic quadratic forms on Hilbert spaces: Application to the eigenvalue problem for compact quadratic forms, Pacific J. Math. (to appear).

2. - A theory of focal points and focal intervals for an elliptic quadratic form on a Hilbert space, Trans. Amer. Math. Soc. 157 (1971), 119-128. 
3. J. Gregory, A theory of numerical approximations for elliptic forms associated with second order differential systems: Application to eigenvalue problems, J. Math. Anal. Appl. (to appear).

4. K. E. Hazard, Index theorems for the problem of Bolza in the calculus of variations, Contributions to the Calculus of Variations, 1938-1941, University of Chicago Press, Chicago, Ill., 1942, pp. 293-356. MR 4, 47.

5. M. R. Hestenes, Applications of the theory of quadratic forms in Hilbert space to the calculus of variations, Pacific J. Math. 1 (1951), 525-581. MR 13, 759.

6. E. Y. Mikami, Focal points in a control problem, Pacific J. Math. 35 (1970), 473-485.

Department of Mathematics, University of California, Los Angeles, CaliFORNIA 90024

Department of Mathematics, University of Alberta, Edmonton, Alberta, Canada (Current address) 